\title{
Glassy with a Chance of Nanophase Iron: Space Weathering of Lunar Soil as Observed with Aberration- Corrected Scanning Transmission Electron Microscopy
}

\author{
K.D. Burgess* and R.M. Stroud \\ U.S. Naval Research Laboratory, 4555 Overlook Ave. SW, Washington, DC 20375 \\ *kate.burgess@nrl.navy.mil
}

\begin{abstract}
Interactions between the space environment and the surfaces of "airless" planetary bodies such as the Moon lead to the formation of complex nano-scale alteration features. State-of-the-art instrumentation and techniques such as aberration-corrected scanning transmission electron microscopy allow for the collection of new data on the structure and composition of these features on lunar dust grains returned during the Apollo missions. The data show a number of unexpected phases present in the grains, such as oxidized iron-rich nanoparticles. The analysis of these particles provides insight into the complex processes that alter the surface of the Moon and other planetary bodies.
\end{abstract}

\section{Introduction}

The Moon, like many other rocky bodies in the solar system such as asteroids and comets, lacks an atmosphere and a strong magnetic field. This means its surface is subject to a number of impact and irradiation processes that do not affect rocks on Earth. Micrometeorites that would burn up before ever reaching the Earth's surface cause fracturing, melting, vaporization, and amorphization on the lunar surface. Charged particles, mostly electrons, protons, and alpha particles, in the solar wind are implanted and backscattered and cause amorphization, sputtering, and secondary electron (SE) emission. These processes, collectively known as space weathering, change the lunar surface on the atomic to sub-micrometer scale but have significant consequences for interpretation of remote sensing data such as albedo (surface reflectivity) and visible to near-infrared (VNIR) reflectance. Lunar regolith, that is, the rocks and rock fragments that cover the surface of the Moon, becomes generally finergrained, more glass-rich, and darker with increasing exposure to the space environment [1]. Mineral-specific absorption bands in the VNIR become attenuated, and the spectrum acquires a red slope, with reflectance higher at longer wavelengths going from visible to near-infrared. Laboratory analyses on returned Apollo samples have shown that these optical effects become stronger in the finest sieved fraction of the soils and that there is a relationship between the optical features associated with space weathering and the presence of nanophase metallic iron $\left(\mathrm{npFe}^{0}\right)$ inclusions in the soil particles as measured by ferromagnetic resonance (FMR) [2]. From the initial FMR data, the $n p \mathrm{pe}^{0}$ inclusions were inferred to be $4-33 \mathrm{~nm}$ in size with very little $\mathrm{Ni}$, indicating they formed through melting or vapor deposition of other lunar material rather than being brought to the Moon by meteorites [3].

Observations of lunar soil grains in a transmission electron microscope (TEM) provided direct evidence of the presence and size range of $n \mathrm{nFe}^{0}$ in soil rims and showed a number of other nano- and microscale features associated with space weathering $[4,5]$. The top $100-200 \mathrm{~nm}$ of most grains were seen to be generally amorphous, often containing significant $\mathrm{npFe}{ }^{0}$ inclusions, but in detail these rims were highly complex, involving multiple layers, vesicles, and significant variations in chemistry (Figure 1). The complexity of features seen across all length scales at which the Moon has been observed, from nanometer to planet-wide, has meant that relating specific processes to specific nanostructures and those in turn to specific optical properties is challenging, and new observations across all scales continue to provide important constraints and relationships.

Lunar samples have now been in hand for over 45 years since their return by the Apollo and Luna missions. Although huge leaps in our understanding of the Moon, its composition, and how it formed and evolved have been made since then, the intervening years have also seen significant advances in analytical instrument design and technology, allowing us to examine samples that have been stored in anticipation of just such advancements. Additionally, a number of new spacecraft have provided new remote sensing data from the lunar surface

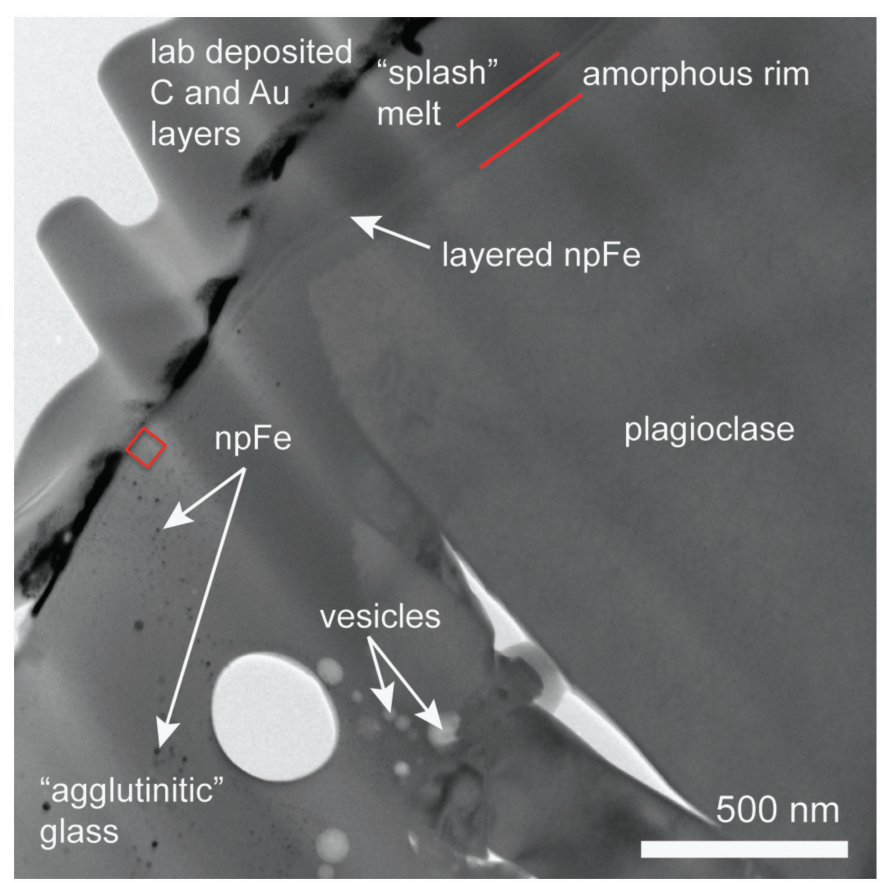

Figure 1: Brightfield STEM image of a plagioclase grain from mature lunar soil 79221 showing many features associated with space weathering. A glassy region with a significant amount of relatively large $\mathrm{npFe}(5-50 \mathrm{~nm})$ covers the left side of the grain. An amorphous rim, composed of several layers with different compositions and abundances of small $\mathrm{npFe}^{\mathrm{x}}(1-6 \mathrm{~nm}$ size range) not visible at this scale but shown in Figure 4 from the area of the red box, is $~ 120 \mathrm{~nm}$ thick and is covered in places by glass of yet another composition, likely a melt splash from a micrometeorite impact on another grain. The image also shows the protective gold and carbon layers deposited in the lab prior to FIB preparation. 


\section{Coated Grids for Cryo TEM from SPI Supplies}

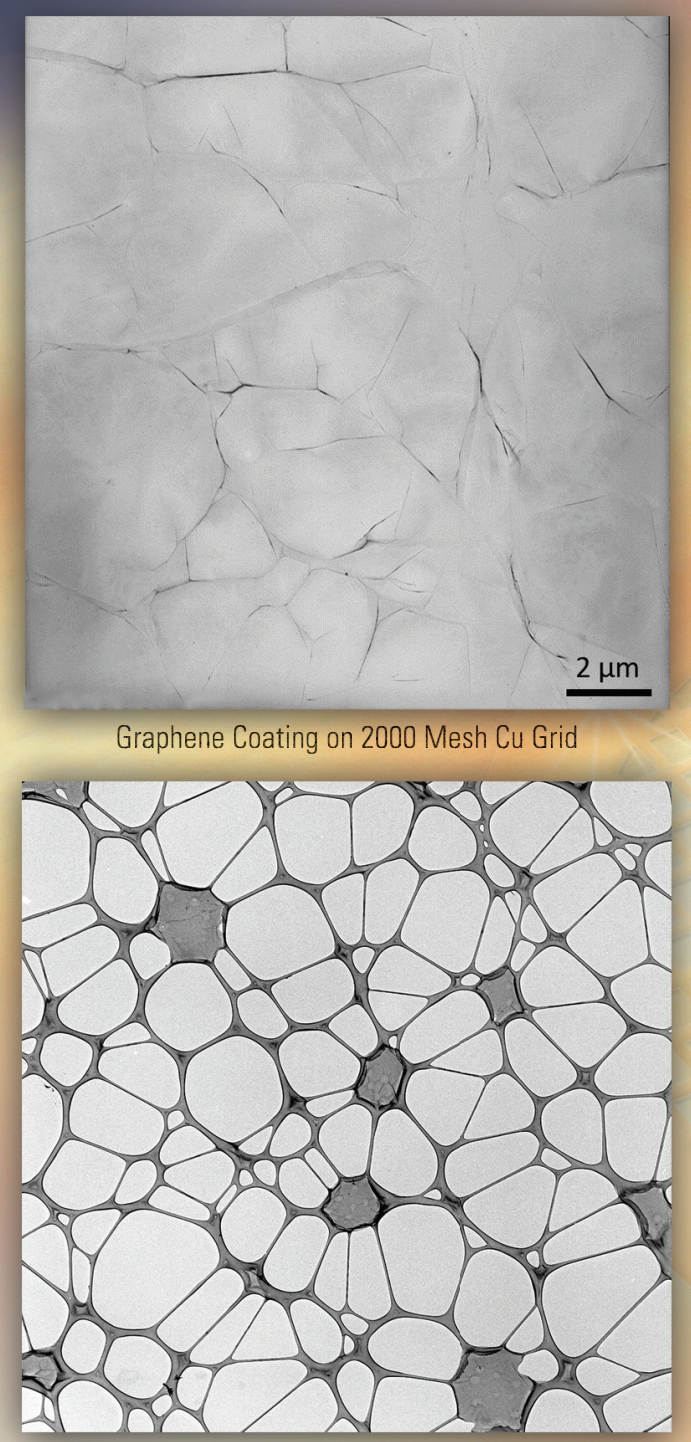

SPI Supplies Brand Lacey Carbon Hilm on Copper Grid
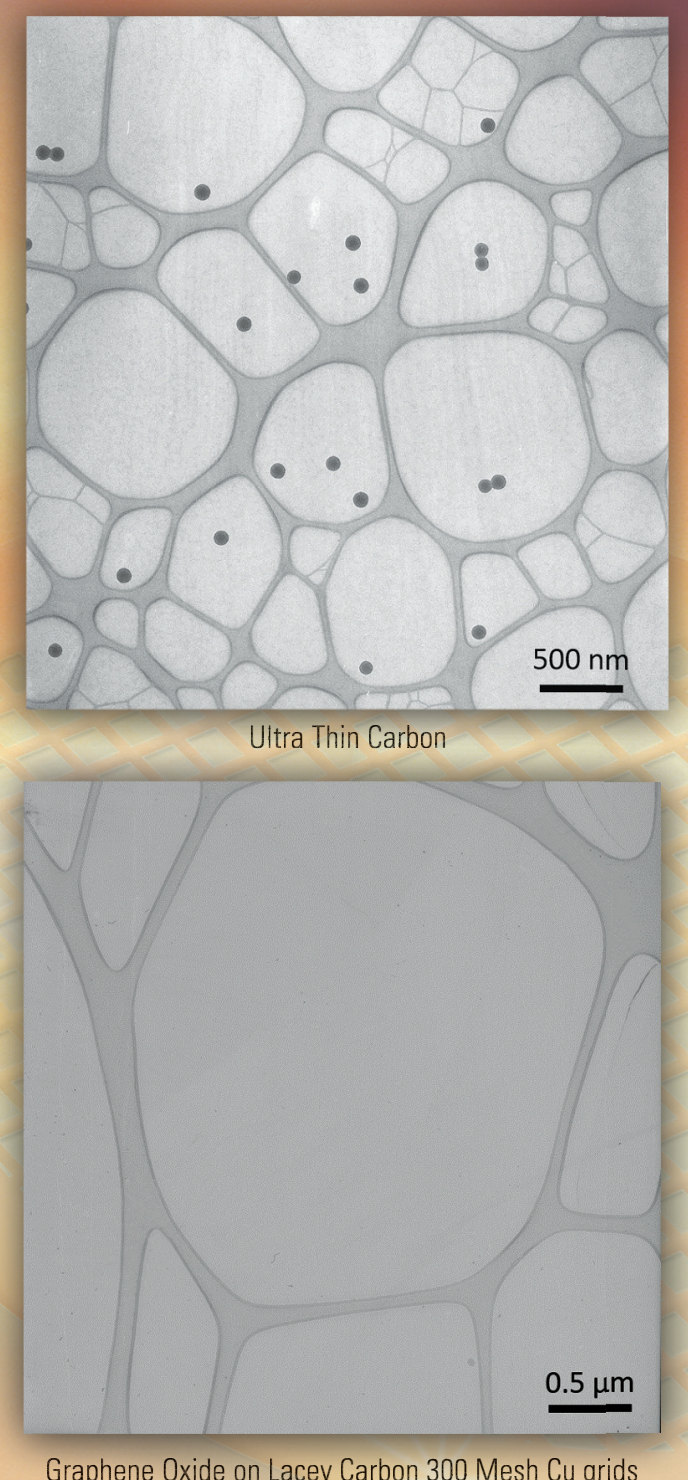

Graphene Oxide on Lacey Carbon 300 Mesh Cu grids

\section{Cryo TEM requires careful selection of the proper grid and support film}

But don't worry - SPI Supplies has you covered. Choose your coating from

traditional carbon, holey carbon, lacey carbon, our new Ultra-Thin carbon, graphene,

or the well-known Quantifoil grids. Need help choosing? Contact us and ask one of our experts.

\section{just a click away... 2spi.com}

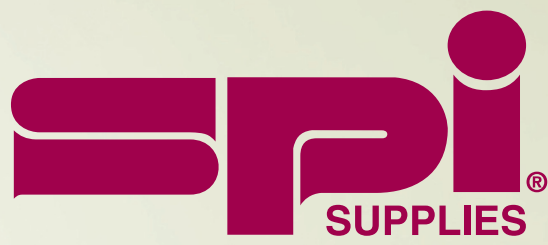

SPI Supplies Division of STRUCTURE PROBE, Inc. 
at spatial and energy resolutions not previously attainable, and new measurements of the lunar exosphere and local magnetic anomalies are increasing our understanding of how the Moon interacts with the space environment. These new instruments and data have shown that the Moon is not "bone dry," as once thought, either in its interior [6] or on its surface [7]. Similarly, recent measurements of iron-rich inclusions in space-weathered rims using aberrationcorrected scanning transmission electron microscopy (acSTEM) have shown the inclusions to be oxidized in some cases [8]. Oxides have different optical characteristics than iron metal, but like water, oxidized material was
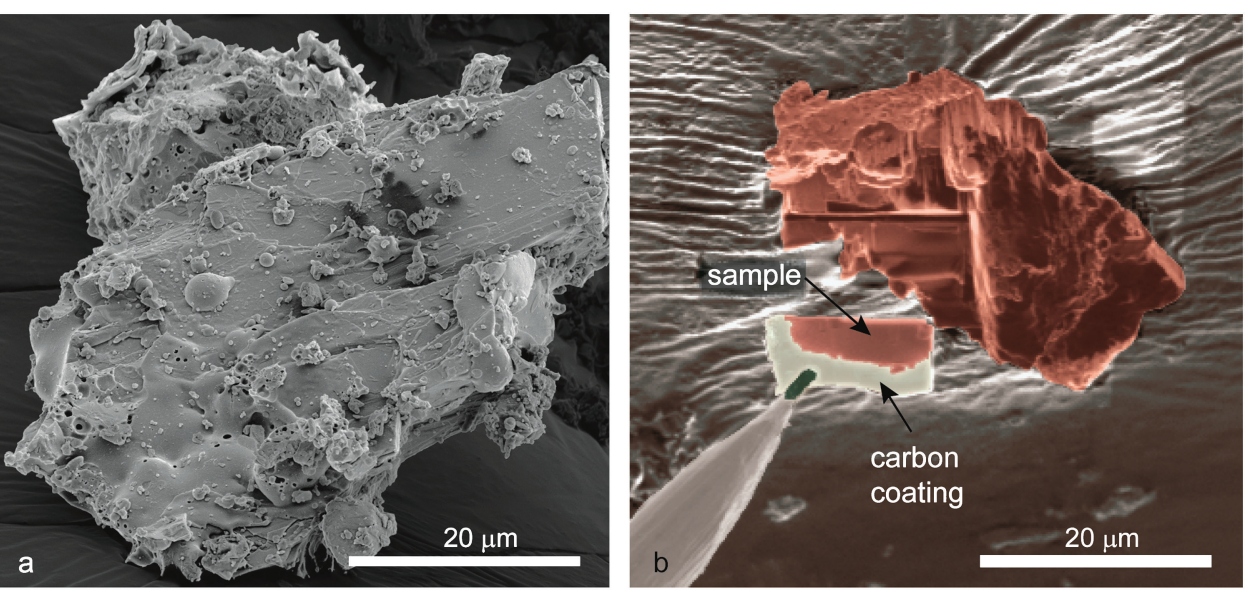

Figure 2: (a) Secondary electron (SE) image of a plagioclase mature soil grain from 79221 coated with a vesicle-rich glass and a number of small mineral and splash melt particles. (b) False-color ion beam-induced SE image of a TEM lift-out sample attached to $\mathrm{W}$ needle after removal from a soil grain (red). An ion beam-deposited $\mathrm{C}$ cap covers the sample to protect it during milling. considered to be very rare on the lunar surface and thus was not included in hyperspectral models or in hypotheses of how space-weathered surfaces evolve. It was only with detailed characterization of individual inclusions that the small but significant fraction of iron oxide nanophase inclusions could be identified.

The work presented in this article uses the combined approach of site-specific focused ion beam (FIB) preparation and acSTEM with both electron energy-loss spectroscopy (EELS) and energy dispersive X-ray spectroscopy (EDS) to further investigate the complex chemistry and nanostructures resulting from space weathering. Previous (S)TEM studies used microtome rather than FIB sample preparation, which can lead to loss of sample context from chattering or fracturing of the silicate soil particles. The combination of multiple analytical techniques at high resolution allows us to continue bridging the gaps in understanding planet-wide processes occurring at the atomic scale.

\section{Materials and Methods}

The samples used here are soils returned from the Moon by the Apollo 17 mission in December 1972. We include three soils with varying degrees of space weathering: mature soil 79221, sub-mature soil 71501, and immature soil 71061 [9]. Since their return, the samples have been stored under dry nitrogen at the Johnson Space Center in Houston, TX. The three soils are high-Ti mare basalts, and aliquots of each soil have been well-characterized optically for bulk chemistry and in terms of their maturities [10]. The soils had been subdivided by grain size, with the smallest size fraction generally consisting of individual mineral or glassy fragments that had been altered by space weathering on almost all surfaces. A small amount of each soil was placed on a sticky carbon film and coated in gold or carbon to prevent charging when viewed in the scanning electron microscope (SEM). From these, we selected 2-3 grains for further focused FIB preparation. Figure 2 shows the surface texture of a mature soil grain and an in-progress FIB lift-out of another grain.

Before milling, the regions of interest were coated with a thick carbon cap $(1-2 \mu \mathrm{m})$, deposited by the ion beam, to prevent damage to the grain surface by the ion beam. The ion

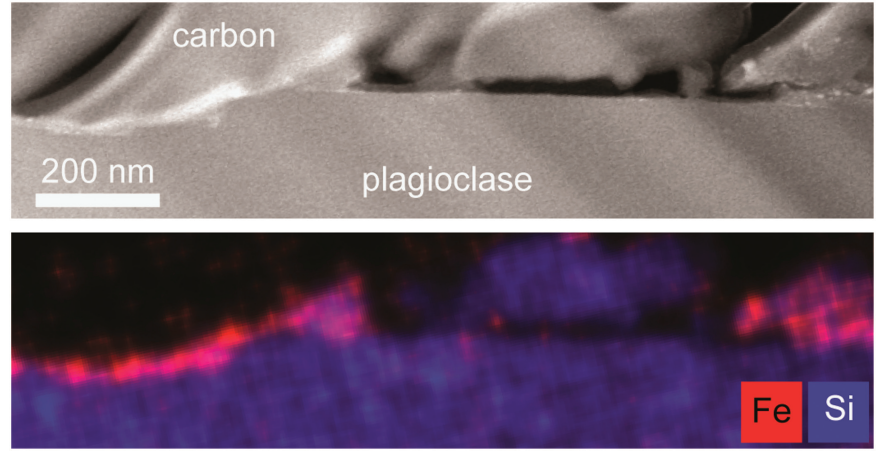

Figure 3: HAADF image (top) and EDS map (bottom) of a plagioclase grain with a carbon deposition rim from immature soil sample 71061. The Fe-containing rim is discontinuous and much thinner than that seen on the mature grain shown in Figure 1. Convergent beam electron diffraction shows there is no amorphous layer in the plagioclase.

beam energy was $30 \mathrm{kV}$, and after the sample was attached to the $\mathrm{Cu}$ half-grid (Pt weld), the sample was thinned to electron transparency. Samples were baked under vacuum at $140^{\circ} \mathrm{C}$ for 8 hours to drive off adsorbed water before analysis in the microscope. Total exposure to the ambient lab atmosphere prior to the first viewing in the STEM was $<1$ hour. One sample was baked and analyzed multiple times, but no systematic differences were seen when comparing data collected on different days.

Analytical data from EELS and EDS were collected on the NION UltraSTEM-X at the U.S. Naval Research Laboratory, which is equipped with a Gatan Enfinium ER EEL spectrometer and a windowless, 0.7 sr Bruker SDD-EDS X-ray detector. The STEM was operated at $200 \mathrm{kV}$ and $\sim 40 \mathrm{pA}$, with a probe size of $0.1-0.2 \mathrm{~nm}$. Data were collected as spectrum images (SI), with a spectrum collected at each pixel for mapping of variations in composition and oxidation state. Moderate dwell times (0.01-0.5s) were used to balance signal-to-noise in low-Fe regions with beam damage effects. Energy dispersions of $0.05 \mathrm{eV} /$ channel or $0.1 \mathrm{eV} /$ channel were used to collect SIs for Fe L-edges and zero-loss peaks (ZLP) during the same scan using Gatan DualEELS capability. The energy resolution 

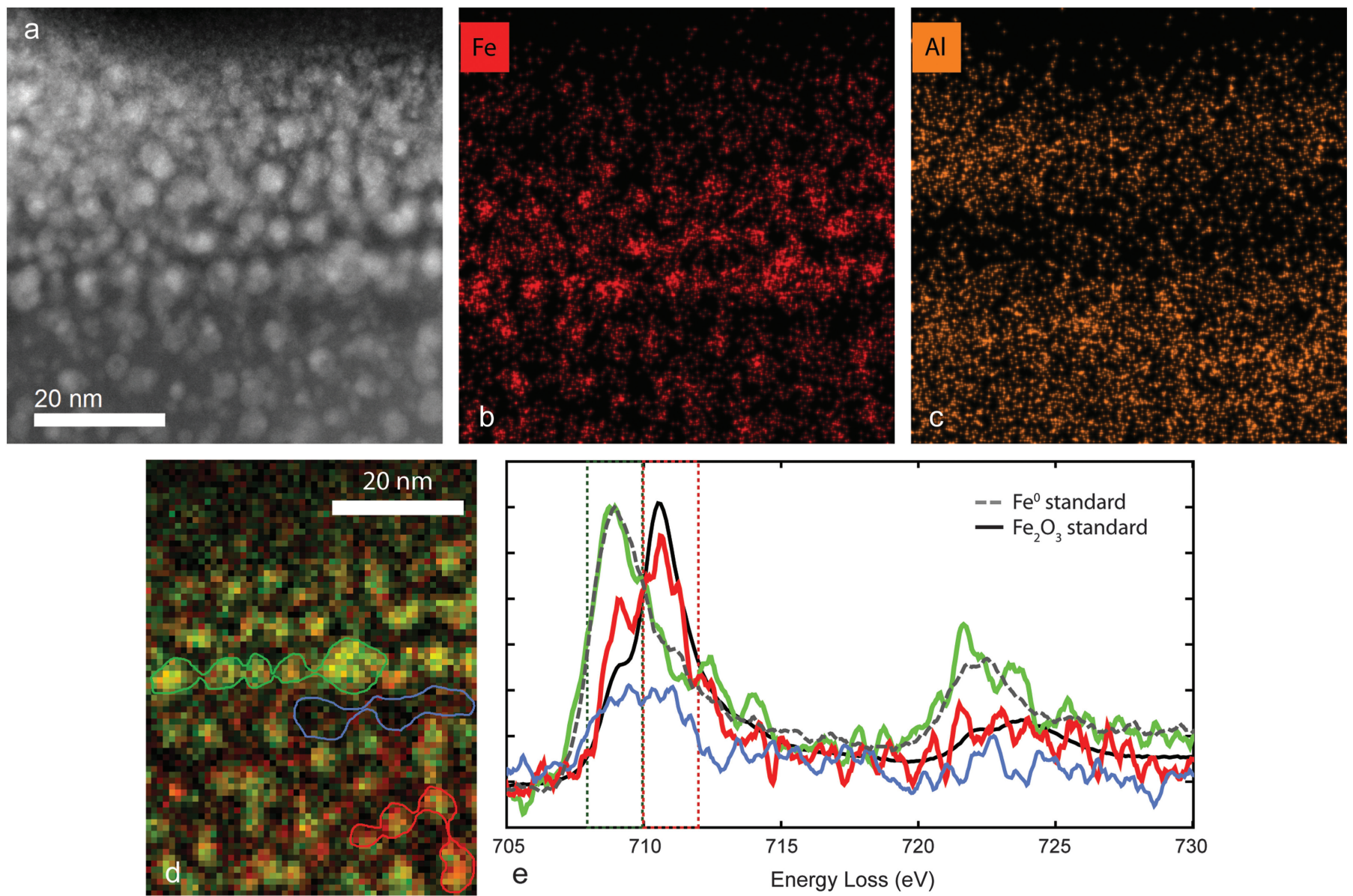

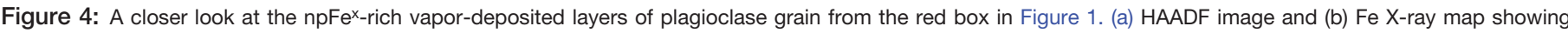

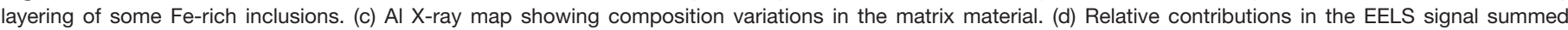

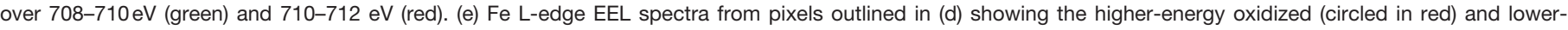

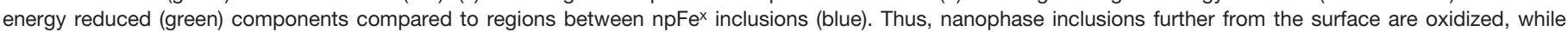

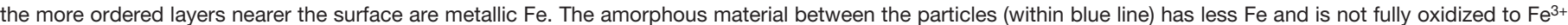
(blue spectrum).

of the SIs, as measured from the ZLP, was $0.4-0.45 \mathrm{eV}$. Peak alignment to compensate for energy drift during SI acquisition was carried out using Gatan Digital Micrograph software to first align the ZLP then apply the same shift at each pixel in the related core-loss SI. The energy of the $\mathrm{Fe}_{2}$ edge shifts between valence states $\mathrm{Fe}^{0}$ and $\mathrm{Fe}^{2+}$, both at $\sim 708 \mathrm{eV}$, and $\mathrm{Fe}^{3+}$ at $\sim 710 \mathrm{eV}$ due to changes in the electronic transitions, allowing an estimation of oxidation state. The EDS data were also acquired as spectrum images; the beam was scanned at a rate of $16 \mu \mathrm{s} /$ pixel for 100-300 frames. Quantification was done using the Cliff-Lorimer method with default $k$-factors, and samples were thin enough to not require an absorption correction.

\section{Results}

Samples thinned by FIB from a number of grains in each soil sample reveal a variety of micro- and nano-structures including glassy "splash" coatings, small mineral grains attached to the surface of larger grains, vapor deposits of varying composition and thickness, vesicles in rims and soil grain interiors, and a number of nanophase inclusions of $\mathrm{Fe}$-metal, $\mathrm{Fe}$-oxide, $\mathrm{Fe}$-sulfide, and ilmenite. The rim morphologies fall into the four general categories described by Keller and
McKay [5]: amorphous, inclusion-rich, multiple, and vesicular. Grains from the immature soil sample have notably thinner or discontinuous rims compared to the sub-mature and mature soils (Figure 3), although one grain from the mature soil also lacks a clear rim and appears much "younger" than other grains from the same sample. Close analysis of some of the nanophase iron-rich inclusions shows variations in oxidation state, both in interior and rim regions. The small number of samples precludes statistics on the abundance of oxidized material, especially as it relates to soil maturity, but oxidized iron-rich inclusions $\left(\mathrm{npFe}^{\mathrm{x}}\right)$ were measured in at least one sample from each soil.

Plagioclase from mature soil. The plagioclase sample shown in Figure 1 from mature soil 79221 has a complex morphology. The soil grain, $\sim 30 \mu \mathrm{m}$ across before FIB preparation, is coated by two different glasses, one rich in iron inclusions and vesicles. The other glass was emplaced on the grain after significant space weathering, and a rim of amorphous plagioclase covered by layers of inclusion-rich amorphous material is seen between the crystalline plagioclase and the inclusion-free glass. Figure 4 shows a close-up of the inclusion-rich layers in a region not covered by the splash melt. The $n p F e^{\mathrm{x}}$ nanoparticles $(1-6 \mathrm{~nm})$ 
apparent in both the HAADF image and Fe X-ray map form layers parallel to the grain surface and to compositional layers, here represented by an Al EDS X-ray map but also evident in $\mathrm{Ca}, \mathrm{Mg}$, and Si maps. Selected EEL spectra from different layers of the $n p \mathrm{Fe}^{\mathrm{x}}$ show that there is also significant variation in oxidation state, with oxidized Fe nanoparticles present in the interior Al-rich layer. The presence of $\mathrm{Fe}^{3+}$ is indicated by the peak at $\sim 710.5 \mathrm{eV}$, whereas $\mathrm{Fe}^{0}$ has a peak at $\sim 708 \mathrm{eV}$. Several of the larger $n p F e^{\mathrm{x}}$ inclusions in the glassy region are also oxidized, although the vast majority are metallic.

Iron sulfide and ilmenite in particle from mature soil. A second grain from soil 79221 is chemically and morphologically very different (Figure 5). Prior to FIB preparation the sample was an almost perfect sphere, $\sim 40 \mu \mathrm{m}$ in diameter; smaller spheres and "splashes" cover the surface, as also seen in Figure 2a, a common feature of mature soils [11]. A glassy "splash" coats much of the surface of the FIB sample and contains significant $\mathrm{npFe}{ }^{0}$; the morphology suggests formation during quench rather than vapor deposition. The interior of the grain is largely devitrified, and most minerals are larger than $500 \mathrm{~nm}$ and were identified using EDS. However, a number of nanocrystals are also observed. Where they are far from other crystals, they are predominately $\mathrm{Fe}^{0}$, but where they are spatially associated with the pyroxene dendrites, Fe-sulfide and ilmenite $\left(\mathrm{FeTiO}_{3}\right)$ are found. Figure 5 shows the context of the sulfide and ilmenite within the larger grain and detailed X-ray maps of $\mathrm{Fe}, \mathrm{Ti}$, and $\mathrm{S}$ showing their close spatial association.

Ilmenite grain in sub-mature soil. Ilmenite responds to space weathering very differently from silicates such as plagioclase. Figure 6 shows images of a dendritic ilmenite grain from sub-mature soil 71501, with intergrowths of chromite, and glassy regions between the limbs of the oxide minerals. Here, the differing responses of the silicate and oxides are apparent. The combination of lattice fringe images, EELS, and EDS show that the ilmenite has elongated $n \mathrm{pFe}^{0}$ inclusions in a defect-rich, nano-crystalline layer that is covered by a thin, amorphous Si-rich layer and an Fe-rich layer (Figure 6b). In contrast to the ilmenite surface, the glassy regions are covered by a layer of spherical $1-5 \mathrm{~nm} n \mathrm{pFe}^{0}$ particles. A larger, pitted core-shell Fe particle is also present at the very surface (Figure $6 \mathrm{c}$ ). EELS analysis shows that the core of this particle, as well as the surrounding smaller $n p \mathrm{Fe}^{0}$, is metallic Fe while the shell has an additional peak at slightly higher energy loss indicating oxidized $\mathrm{Fe}^{3+}$ (Figures $6 \mathrm{c}$ and $6 \mathrm{~d}$ ). Lattice fringes from near the center of the particle and closer to the edge, where the shell is a larger proportion of the material, are consistent with Fe metal and magnetite, respectively.

\section{Discussion}

The combination of spectroscopic data from natural and analog lunar material with analytical (S)TEM measurements have greatly aided the interpretation of remote sensing data while demonstrating the variability among soil particles even within the same sample. The complexity of the nanostructures and the variations in composition and oxidation state in the nanophase Fe-rich inclusions shown here make linking specific optical characteristics in the bulk material, such as slope changes
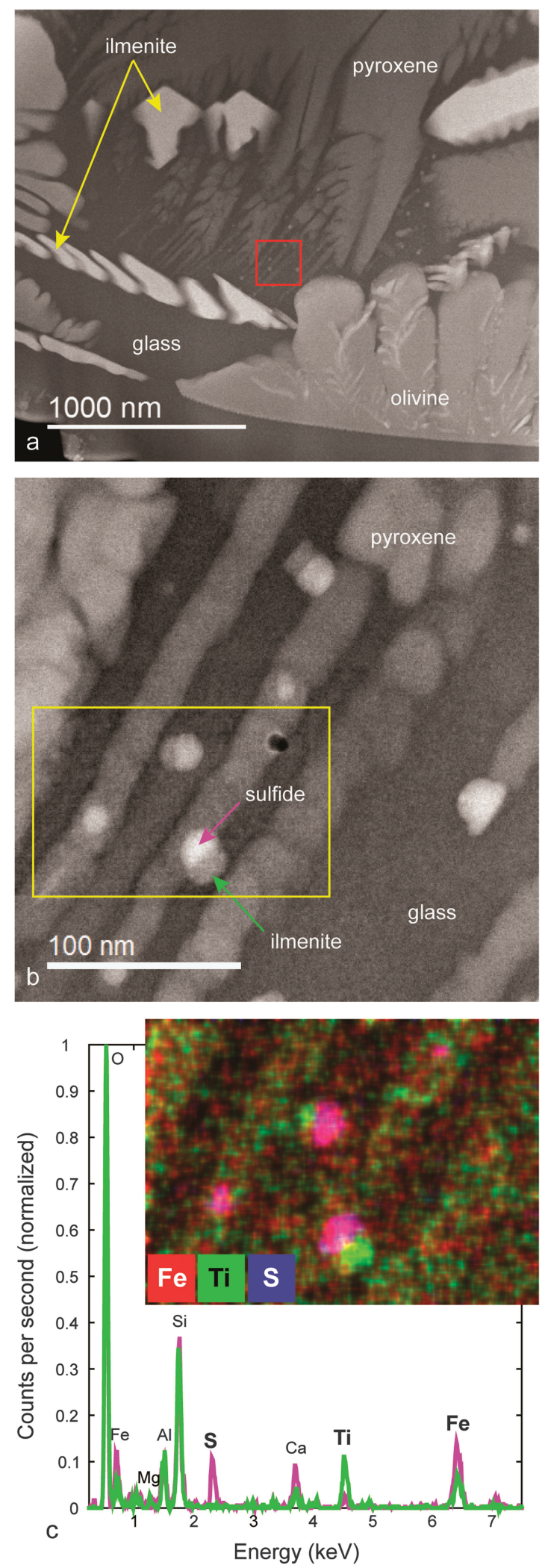

Figure 5: (a) HAADF image of a multiphase, mineralogically complex soil particle from mature soil 79221. The minerals present in the quenched glass (ilmenite, olivine, pyroxene) are common in the bulk material and were identified here using EDS. (b) At higher magnification, Fe-rich nanocrystals are observed in glassy regions surrounding pyroxene dendrites. (c) EDS analysis shows the nanocrystals are $\mathrm{Fe}$-sulfide and ilmenite $\left(\mathrm{FeTiO}_{3}\right)$, often in close spatial association. The inset EDS map of $\mathrm{Fe}, \mathrm{Ti}$, and $\mathrm{S}$ shows two multiphase particles. 

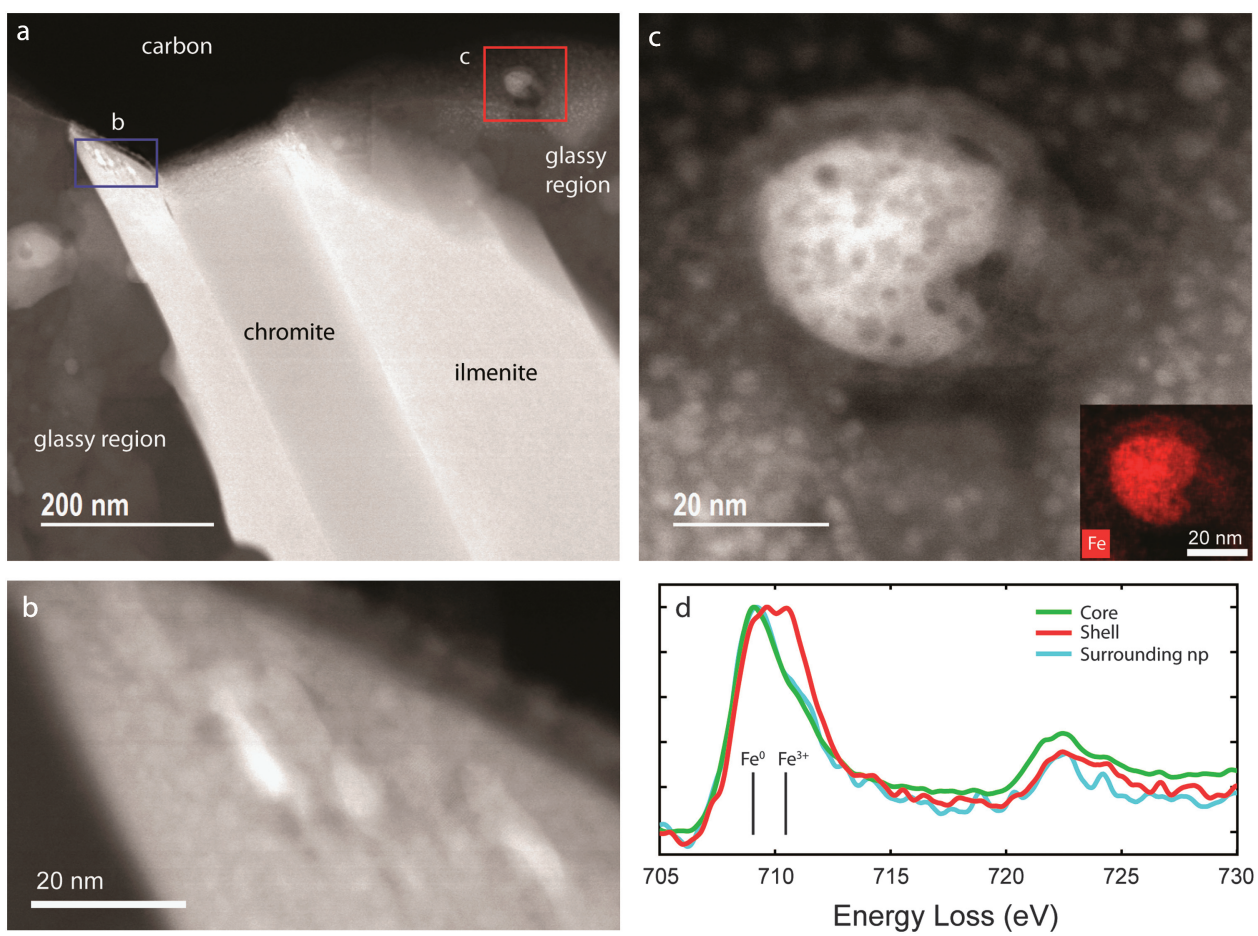

Figure 6: (a) HAADF image of limb of dendritic ilmenite $\left(\mathrm{FeTiO}_{3}\right)$ and chromite grain with glassy matrix showing differences in space weathering features compared to plagioclase in Figures 1 and 3. (b) A closer view of the ilmenite surface showing elongated $\mathrm{npFe}{ }^{0}$, confirmed via EELS and lattice fringe images, and multiple layers that have variable composition (blue box in (a)). (c) Image of the core-shell Fe-rich nanoparticle and smaller npFe ${ }^{0}$ inclusions on the grain surface (red box in (a)). (inset) EDS shows the shell contains significant Fe, although not as much proportionally as the core. (d) EELS analysis supports this since the core region is predominantly metallic Fe (green), similar to the much smaller $\mathrm{npFe} \mathrm{e}^{0}$ inclusions (blue), whereas the shell of the large $\mathrm{npFe} \mathrm{e}^{\mathrm{x}}$ contains significant oxidized Fe in addition to the signal from the surrounding metallic inclusions, leading to a broad peak shifted to slightly higher energy (red).

over specific wavelength ranges, to individual space weathering features challenging. However, these observations can guide experimental preparation of analog material and the choice of end-members used in modeling reflectance and absorption bands in spectral measurements [12]. The observations of nanophase oxide and sulfide inclusions in these soils indicates that their optical parameters may be important in interpretation of remote sensing data from areas such as the polar regions [13] and lunar swirls [14] or local magnetic anomalies, which have anomalous albedo compared to surrounding regions. The preferred orientation of elongated $\mathrm{npFe} \mathrm{e}^{0}$ inclusions in ilmenite could also affect interpretations of inclusion sizes.

The observations of oxidized $n p F e^{\mathrm{x}}$ layers (Figure 4) as well as individual oxidized $\mathrm{npFe}^{\mathrm{x}}$ (Figure 6) indicates that oxidation may occur via several different pathways. Soil particles may become locally oxidized because of excess oxygen from the sputtering of cations or through degassing of $\mathrm{H}_{2}$ following implantation of $\mathrm{H}^{+}$, secondary space weathering processes not often taken into consideration. Thompson et al. [8] noted the presence of hollow oxidized $n \mathrm{nFe}^{\mathrm{x}}$ embedded in the amorphous silicate matrix, an indication that some of the $n \mathrm{pFe}^{\mathrm{x}}$ are oxidized via the Kirkendall effect, where the bonding of $\mathrm{O}$ on the surface of the metallic grain leads to outward Fe diffusion balanced by vacancy diffusion into the metallic particle. The vacancies coalesce and a hollow sphere of oxidized material forms. The core-shell particle with multiple pores shown in Figure 5 supports this hypothesis. In other cases we have observed metallic $n \mathrm{pFe}^{0}$ inclusions that appear pitted and rough much like the core of this particle but have no shell of oxidized material distinguishable from the matrix. The pitting and corroded look of $n \mathrm{PFe}^{0}$ inclusions is likely due to surface diffusion and local driving forces, as $\mathrm{Fe}$ on the inclusion surface is oxidized to $\mathrm{Fe}^{2+}$ and diffuses away into the surrounding silicate matrix.

The limited number of lunar samples available for investigation in terrestrial laboratories means that each sample is highly valuable and care should be taken to gather as much information as possible from each sample. The combined use of acSTEM imaging and EELS and EDS spectrum images provides a powerful dataset but comes with significant challenges. Amorphous silicates are susceptible to electron beam damage leading to oxidation and diffusion $[15,16]$. We attempted to mitigate this damage as much as possible by using fast scan rates. We are also able to account for possible damage effects by repeating EELS measurements for comparison over time and having spectra from not only $\mathrm{npFe} \mathrm{e}^{\mathrm{x}}$ inclusions but also surrounding material. Spectra from matrix material often show it to be oxidized but have much less Fe than the inclusions, and, as shown in Figure 4, the oxidized inclusions are more oxidized than the matrix. We also see that the $n p F e^{0}$ inclusions do not change spectrally or morphologically during measurement.

\section{Conclusion}

As technique and instrument developments allow us to measure the composition and oxidation state of space-weathered materials at finer scales, new complexity in nanostructures can be observed. The wide range in features seen in each of these lunar soils emphasizes the importance of nano-scale observations of a large number of grains in order to gain understanding of space weathering processes, time evolution, and differences among airless bodies. The materials observed here show that oxidized nanophase $\mathrm{Fe}$, both in vapor-deposited rims and grain interiors, could be an important phase contributing to the optical properties of space-weathered surfaces. Loss of hydrogen during heating or movement of oxygen in the silicate matrix as new material is sputtered, both of which could lead to oxidation of $\mathrm{nFe}^{0}$, need to be considered among the many complex processes that contribute to the alteration of these surfaces. Examination of various nanophases other than metallic Fe may be needed to fully account for space weathering features seen in remote measurements. 


\section{Acknowledgements}

This work was supported by the Remote, In Situ, and Synchrotron Studies for Science and Exploration (RIS $\left.{ }^{4} \mathrm{E}\right)$ node of NASA's Solar System Explorations Research Virtual Institute (SSERVI). This is SSERVI publication SSERVI-2017-056.

\section{References}

[1] P Lucey et al., Rev Min Geochem 60(1) (2006) 83-219.

[2] SK Noble et al., M\&PS 36(1) (2001) 31-42.

[3] RM Housley et al., LPSC 4 (1973) 2737-49.

[4] LP Keller and DS McKay, Science 261(5126) (1993) 1305-07.

[5] LP Keller and DS McKay, GCA 61(11) (1997) 2331-41.

[6] AE Saal et al., Nature 454(7201) (2008) 192-95.

[7] CM Pieters et al., Science 326(5952) (2009) 568-72.

[8] MS Thompson et al., M\&PS 51(6) (2016) 1082-95.

[9] RV Morris LPSC 9 (1978) 2287-97.

[10] LA Taylor et al., JGR 106(E11) (2001) 27985-99.

[11] SJ Wentworth et al., M\&PS 34(4) (1999) 593-603.

[12] B Hapke, JGR 106(E5) (2001) 10039-73.

[13] PG Lucey et al., JGR 119(7) (2014) 1665-79.

[14] DT Blewett et al., JGR 116(E2) (2011).

[15] LAJ Garvie et al., Am Min 89(11-12) (2004) 1610-16. [16] KD Burgess et al., Am Min 101(12) (2016) 2677-88.

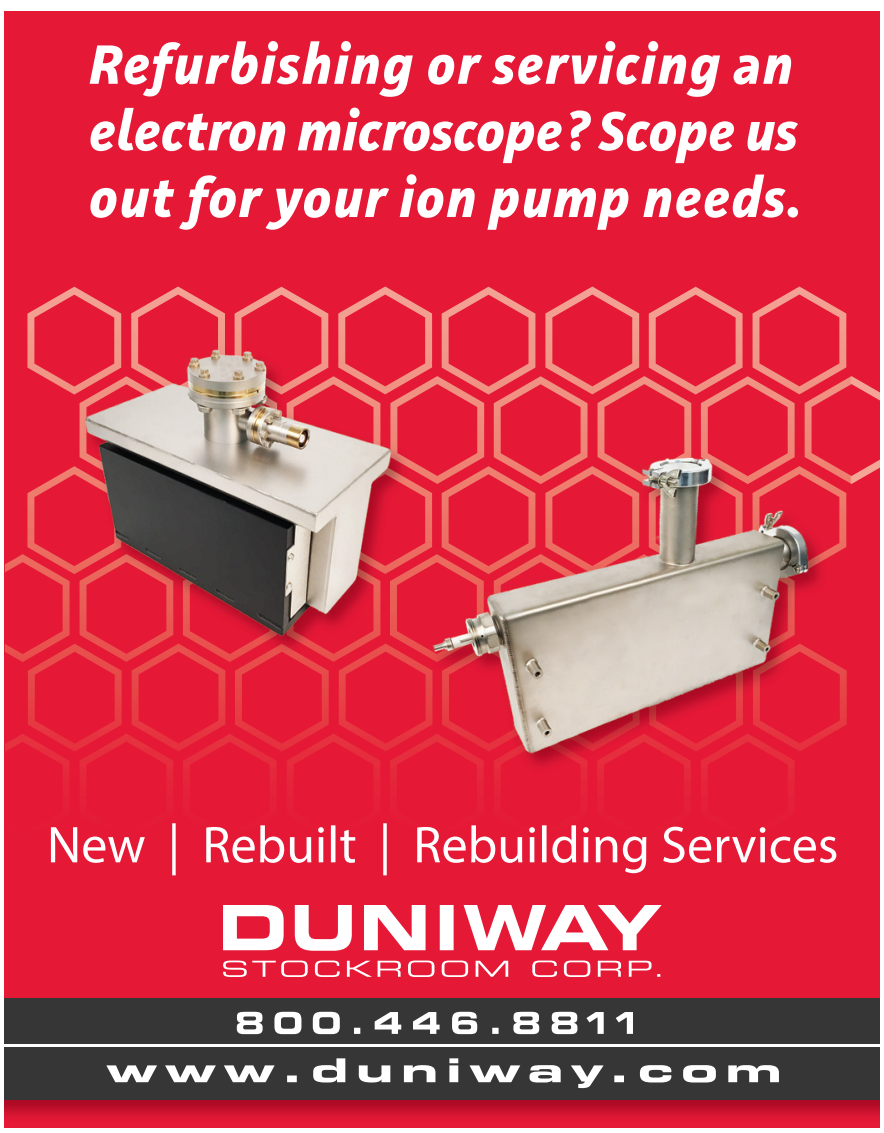

\section{Fast Pump Down with Evactron ${ }^{\circledR}$ \\ Cleaning}

- Evactron Turbo-Plasma ${ }^{\mathrm{TM}}$ cleans your entire system in less than $\mathbf{5}$ minutes.

- Maintain a pristine clean chamber as proven by RGA analysis*.

- Reach base pressure faster.

- Obtain best analytical data with decreased noise.

- Increase daily throughput and reduce costs.

- Push-button recipe selection

- RP, TP and UHV designs

The Fastest Way to Pristine Evactron ${ }^{\circledR}$

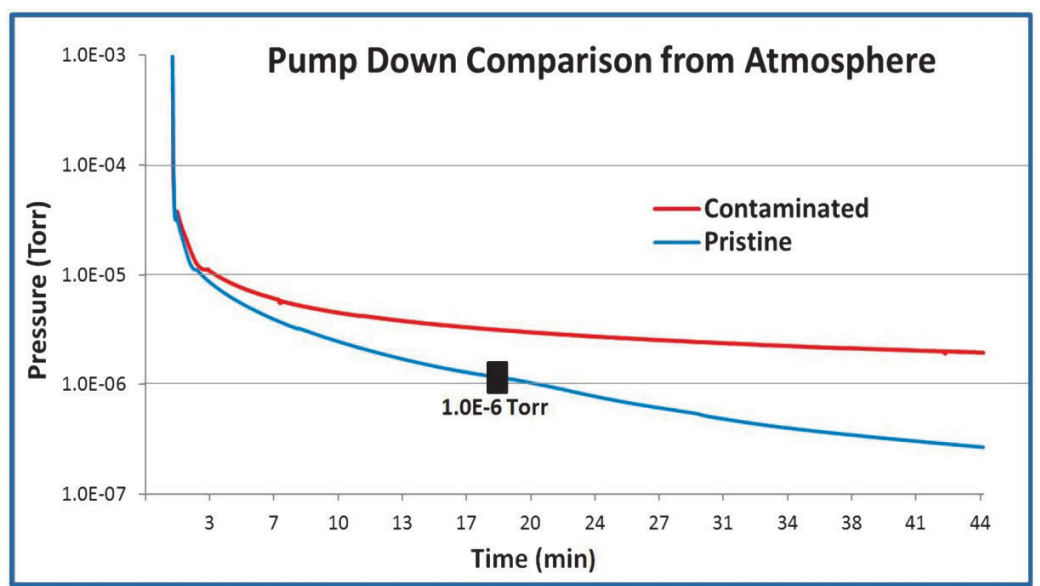

${ }^{*} R G A$ data, cleaning data, and conditions to be found at www.evactron.com.
WWW.EVACTRON.COM 1-650-369-0133 


\section{lumencor}

YE会RS

light for life sciences

light engines for a

\section{BRIGHTER. GREENER. PLANET.}

\section{ANNUAL EARTH DAY PROMOTION}

10 FREE "like new"

SOLA SM or SOLA SE light engines with minimum purchase while supplies last.

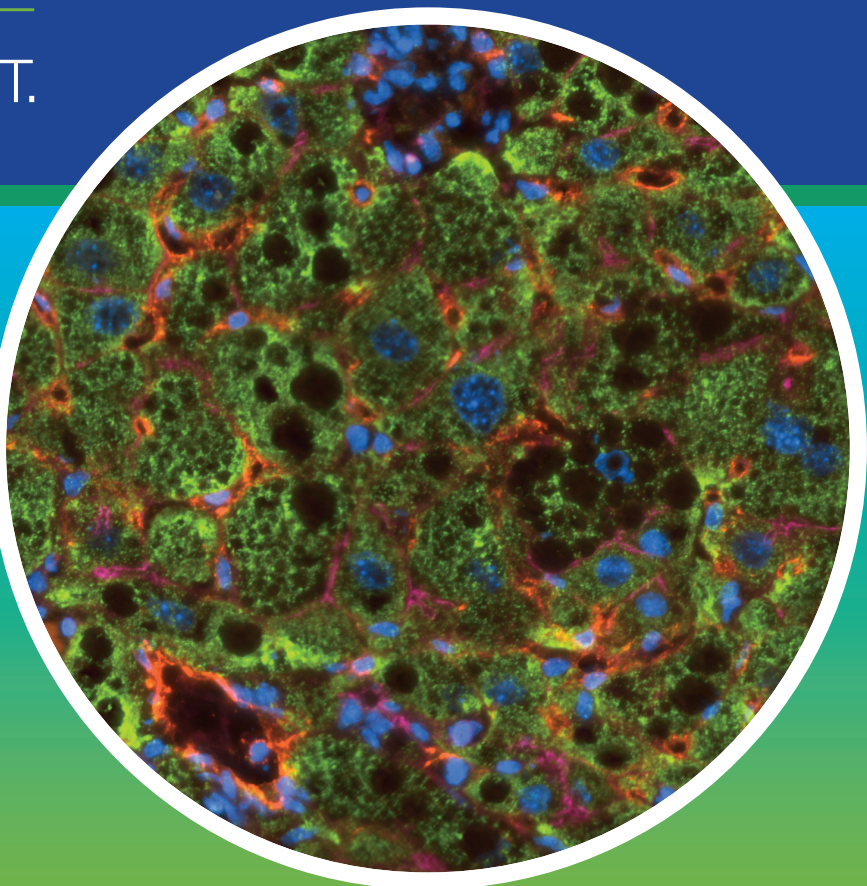

\section{SOLA SM or SOLA SE light engine ${ }^{\circledR}$}

\section{Mercury Free, Solid State Lamp}

- Simple, convenient, solid state arc lamp replacement

- Bright, full spectrum, white light for fluorescence microscopy

- Single button on/off operation or electronic control

- Electronic on/off and intensity control available in SOLA SE model

- Compatible with all major fluorescence microscope models

- Cool, low energy consumption for years of robust use

- Mercury free, maintenance free, service free

- One light engine per customer with qualifying purchase

- $\$ 5,000$ - \$7,500 list price value
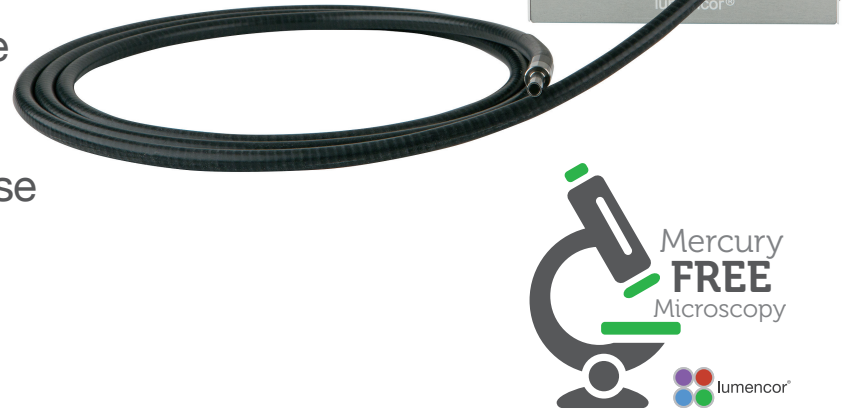